\title{
The interacting binary V 393 Scorpii: another clue for Double Periodic Variables
}

\author{
Ronald Mennickent $^{1}$, Zbigniew Kołaczkowski ${ }^{1,2}$, Gojko Djurasevic ${ }^{3}$, \\ Gabriela Michalska ${ }^{1}$ and Daniela Barría ${ }^{1}$ \\ ${ }^{1}$ Departamento de Astronomía, Universidad de Concepción, Chile \\ email: rmennick@udec.cl \\ ${ }^{2}$ Instytut Astronomiczny Uniwersytetu Wroclawskiego, Wroclaw, Poland \\ ${ }^{3}$ Astronomical Observatory, Belgrade, Serbia
}

\begin{abstract}
We give a brief report on spectroscopic properties of V 393 Scorpii. H $\alpha$ emission and shape and radial velocity of HeI 5875 are modulated with the long cycle. The long cycle is explained as a relaxation cycle in the circumprimary disc, that cumulates the mass transferred from the donor until certain instability produces disc depletion.
\end{abstract}

Keywords. binaries: mass loss, evolution.

\section{Introduction}

After the discovery of a long periodicity in the V 393 Sco ASAS light curve by Pilecki \& Szczygiel (2007), we realized that this star is a bright Galactic member of the new class of interacting binaries Double Periodic Variables (DPVs), that are characterized by two closely related photometric periodicities (Mennickent et al. 2003, 2009c). They have been interpreted as intermediate mass semi-detached binaries experiencing cyclic mass loss into the interstellar medium (Mennickent et al. 2008, Mennickent \& Kolaczkowski 2009 a,b,c). The mechanisms for cyclic mass loss still is a matter of research, and a study of V393 Sco could yield important insigths on the DPV phenomenon.

\section{Observations}

During the last two years we have monitored V 393 Sco with several high resolution spectrographs (e.g. CORALIE, UVES, FEROS, CRIRES) covering several orbital cycles and a couple of long cycles. We have also used the published ASAS V-band light curve and a special code (Djurasevic et al. 2008, 2009) for modeling the system.

\section{Results}

Spectral lines of the donor are clearly visible and follow the orbital motion without interference during the long cycle, but the gainer (primary) is apparently (at least partially) hidden by a circumprimary disc and He I absorption lines follow peculiar motions during orbital/long cycles (Fig. 1). The $\mathrm{H} \alpha$ double emission indicates an outer optically thin part of the circumprimary disc. A Feros spectrum obtained at orbital phase 0.00 and long phase 0.94 shows $\mathrm{H} \alpha$ emission much stronger than a UVES spectrum taken at orbital phase 0.97 and long phase 0.57 (Fig. 1). This implies that the $\mathrm{H} \alpha$ emission (eventually the disc) is larger on the long cycle maximum. The central absorption deeper than the continuum at this epoch suggests that the disc is eclipsed by the donor. If we interpret the HeI absorption lines as produced in a deeper, optically thicker disc region, 

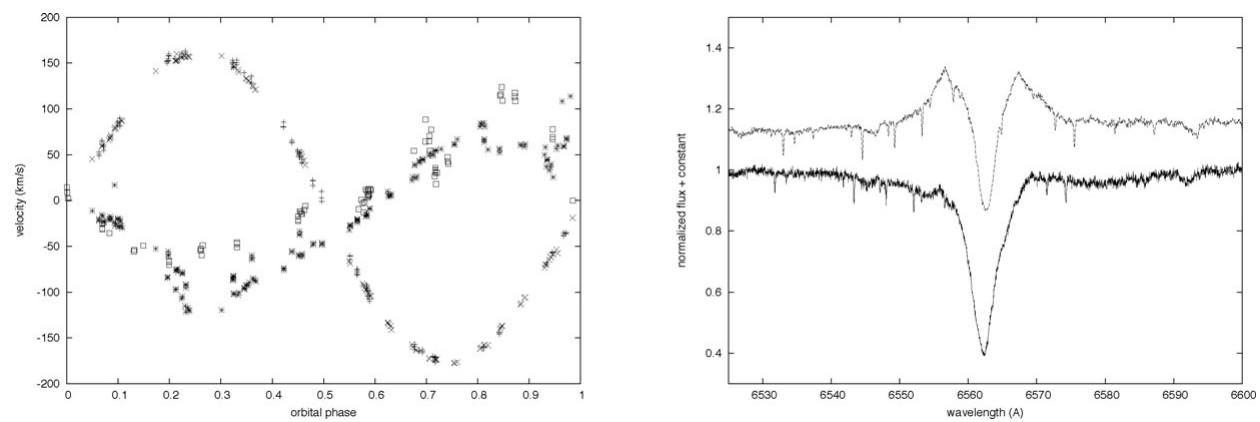

Figure 1. (Left) Radial velocities of the donor star yield $K_{2}=169.7 \pm 0.6 \mathrm{~km} / \mathrm{s}$ (crosses and pluses) whereas HeI 5875 radial velocities at different long cycle phases, $0.2<\Phi_{l} \leqslant 0.8$ (asterisks) and $0.8<\Phi_{l} \leqslant 0.2$ (squares), are peculiar. Orbital ephemeris is from Kreiner (2004). (Right) $\mathrm{H} \alpha$ emission at $\Phi_{l}=0.94\left(\Phi_{o}=0.00\right.$, above) and $\Phi_{l}=0.57\left(\Phi_{o}=0.97\right.$, down $)$.

their RVs indicate strong changes in the structure of this region during the long cycle. The presence of strong line absorption wings at specific phases at the He I $1083.3 \mathrm{~nm}$ line indicates mass exchange/loss in the system. The strong modulation of the FWHM for the He I 5875 line during long cycle could reflect a rotational instability of the circumprimary disc (Mennickent et al. 2009c). He I 6678 is strongly influenced by emission shoulders and does not show this behavior. A comparison with a grid of spectra from NLTE atmosphere models provided by Dr. Ewa Niemczura yields $T_{2}=7900 \pm 100 \mathrm{~K}$ and $\log g_{2}=3.0 \pm 0.1$. It is difficult to estimate the mass ratio, since the helium lines, that could be indicators of the primary motion, do not show a consistent value for $K_{1}$. However, the best fit to the light curve of V 393 Sco always requires the presence of a circumprimary disc under reasonable assumptions. Using $q=0.25$ and $q=0.41$ as trials we obtain $T_{1}=16910 \pm 150$ $K$ and $T_{1}=17400 \pm 300 K$, respectively. These figures agree with the spectral type for the primary we derive from an analysis of archival IUE spectra, viz. B2-3 III. A detailed study of V 393 Sco is in preparation.

\section{Acknowledgement}

We acknowledge support by projects Fondecyt 1070705, Fondecyt 3085010, FONDAP 15010003, BASAL PFB-06, MECESUP and Sociedad Chilena de Astronomía.

\section{References}

Djurašević, G., Vince, I., \& Atanacković, O. 2008, AJ, 136, 767

Djurašević, G., Vince, I., Khruzina, T. S., \& Rovithis-Livaniou, E. 2009, MNRAS, 396, 1553

Kreiner, J. M. 2004, Acta Astronomica, 54, 207

Mennickent, R. E., Pietrzyński, G., Diaz, M., \& Gieren, W. 2003, A\&A, 399, L47

Mennickent, R. E., Kołaczkowski, Z., Michalska, G., Pietrzyński, G., Gallardo, R., Cidale, L., Granada, A., \& Gieren, W. 2008, MNRAS, 389, 1605

Mennickent, R. E. \& Kolaczkowski, Z. 2009a, Revista Mexicana de Astronomia y Astrofisica Conference Series, 35, 166

Mennickent, R. E. \& Kołaczkowski, Z. 2009b, arXiv:0904.1539

Mennickent, R. E. \& Kołaczkowski, Z. 2009c, arXiv:0908.3900

Pilecki, B. \& Szczygiel, D. M. 2007, Information Bulletin on Variable Stars, 5768, 1 